\title{
MicroRNAs Challenge the Status Quo of Therapeutic Targeting
}

\author{
Danish Sayed $\cdot$ Shweta Rane $\cdot$ Maha Abdellatif
}

Received: 1 August 2008 / Accepted: 19 August 2008 / Published online: 9 September 2008

(C) The Author(s) 2008. This article is published with open access at Springerlink.com

\begin{abstract}
MicroRNAs (miRNAs) are recently discovered posttranscriptional regulators of gene expression that have become a cause célèbre. There are currently more than 600 miRNAs identified in humans that are estimated to regulate about one third of all messenger RNA (mRNA). Because miRNA levels were found widely deregulated in diseases, they have been implicated in the underlying pathogenesis. In addition, the changes in their expression patterns are proving to be reliable diagnostic and prognostic measures. But the specific mRNA targets and, hence, function of each miRNA is still work-in-progress. This information would be necessary before fully exploiting miRNA for therapeutic purposes. In this review we will discuss why miRNAs are considered major posttranscriptional regulators and how they impact gene expression and cell function during cardiac hypertrophy and failure. In addition, we will highlight their potential for therapeutic targeting.
\end{abstract}

Keywords MicroRNA - Posttranscriptional Regulation · miRNA Eraser $\cdot \mathrm{miR}-1 \cdot \mathrm{miR}-133 \mathrm{a} \cdot \mathrm{miR}-21$.

Therapeutic Targeting

\section{MicroRNA and the Impact of Posttranscriptional Regulation on Gene Expression}

MicroRNAs (miRNAs) are short oligoribonucleotides that posttranscriptionally regulate gene expression. Guided by sequence complementarity, they specifically target messenger RNA (mRNA) and inhibit translation or induce degradation.

D. Sayed $\cdot$ S. Rane $\cdot$ M. Abdellatif $(\bowtie)$

Cardiovascular Research Institute,

Department of Cell Biology and Molecular Medicine,

University of Medicine and Dentistry of New Jersey,

Newark, NJ 07103, USA

e-mail: abdellma@umdnj.edu
Posttranscriptional regulatory mechanisms appear to play a fundamental role in determining a gene's translational output. This is deduced from the simple fact that relative mRNA levels do not reflect the corresponding cellular protein levels [1]. A study by Beyer et al. confirmed weak or no correlation between mRNA and protein abundance for the whole cell [2]. Similar results were obtained for measurements of different cellular compartments. But, interestingly, a more positive correlation emerged when they grouped functionally related genes. In agreement, the decay rates of mRNA for genes that are functionally related were similar [3]. This suggests that functionally related genes are posttanscriptionally regulated by a common denominator.

According to our current knowledge of miRNAs and their targets, an individual miRNA has the potential to regulate a set of specific genes involved in a common cellular function. For example, we observed that miR-1 targets an array of growth-related genes that have been previously implicated in the development of cardiac hypertrophy, which include Ras GTPase-activating protein, cyclin-dependent kinase 9, endothelin, fibronectin, Ras homologue enriched in brain, eukaryotic initiation factor $4 \mathrm{E}$, JunD, quaking, insulin-like growth factor, and Rap1. In accordance, upon induction of cardiac hypertrophic growth, miR-1 is downregulated and translation of those targets is uninhibited [4]. Similarly, when John et al. [5] analyzed individual miRNA for gene ontology they observed that some favored certain terms. For example, miR-208 targets were biased toward "transcription factor," while miR-105 had a preponderance of "small GTPase-mediated signal transduction" [5]. Thus, miRNAs offer a mechanism whereby functionally related genes can have similar expression patterns and decay rates.

Farh et al. reported that predicted mRNA targets of tissue-enriched miRNAs were lower in the corresponding tissue [6]. Moreover, those targets were initially high in those tissues before the increase in the targeting miRNA, 
albeit they were not completely eliminated. This suggested that miRNAs are involved in both mRNA decay, as well as, translational repression. In agreement, Lim et al. showed that by expressing the muscle-specific miR-1 or the brainspecific miR-124 in HeLa cells the mRNA expression pattern shifted towards the corresponding tissue [7]. These data support the view that posttranscriptional regulation by miRNA defines tissue function and fate.

\section{Evidence that miRNA are Major Regulators of Gene Expression and Function in the Heart}

Similar to other pathologies, cardiac hypertrophy and failure are characterized by an overall modification of the gene expression profile within the cardiac myocytes. During compensatory hypertrophy this profile appears, to a great extent, to recapitulate that of the neonatal heart. This is in concordance with the fact that the latter is also in a phase of hypertrophic growth [8]. Conceivably, this switch is triggered by transcriptional and/or posttranscriptional events. If we assume the position that miRNA play a major role in the reprogramming of gene expression during hypertrophy we, thus, are predicting that miRNAs expression will, in parallel with its targets, revert to the neonatal pattern. We tested this hypothesis, and as seen in Fig. 1, the majority of miRNAs that were differentially deregulated in pressure overload hypertrophy vs. sham hearts in a mouse model reverted to their neonatal vs. adult pattern. These results provided us with the first clue that miRNA play a major role in gene regulation during cardiac hypertrophy.

MiR-1 is one of the better-characterized miRNA, which constitutes $\sim 45 \%$ of total miRNA in the heart. First identified as a muscle-enriched miRNA [9], its expression is detected as early as embryonic day (E) 8.5 in the mouse heart, and increases with the progression of differentiation [10]. In Drosophila, a DmiR-1 ${ }^{\mathrm{KO}}$ mutant dies as a small second instar larvae, 2-7 days after hatching, from apparent paralysis [11]. Similarly, disrupting miR-1-2 in a homozygous mouse model results in ventricular septal defects and lethality between E15.5 to immediate postnatal [12]. Recently, a few reports have also implicated miR-1 in development of cardiac hypertrophy [4, 13] and arrhythmias [14]. This further proves that miRNA are major regulators of gene expression that govern heart development and function.

\section{MiRNA Mediate Specific Translation Regulation "On Demand"}

Although, there are many known posttranscriptional regulators of gene expression, there are none that afford such a global and specific effect as miRNAs, which is why they

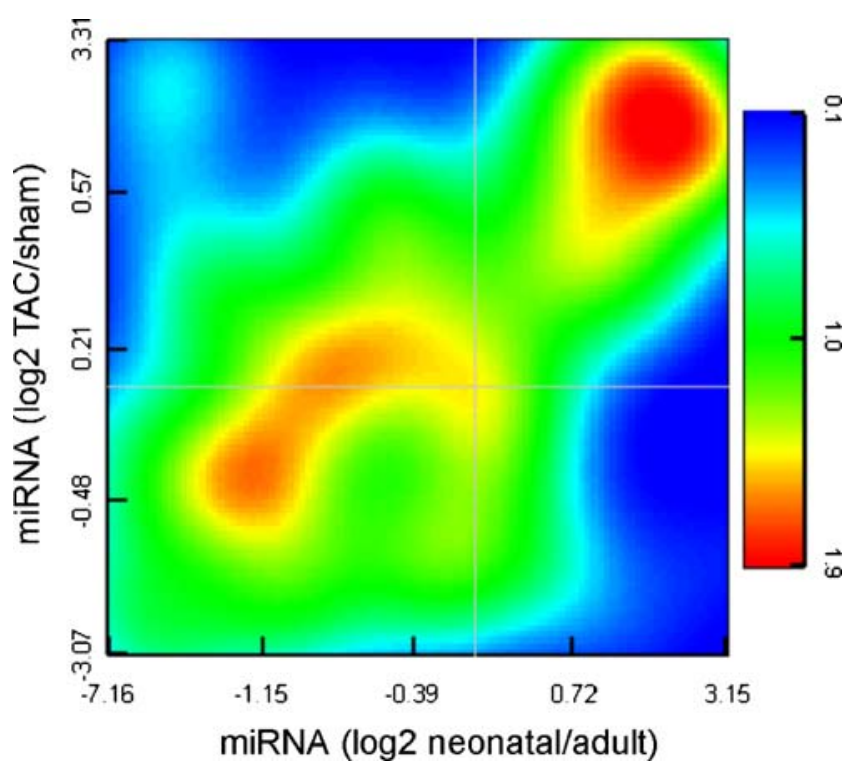

Fig. 1 Comparison between the differentially expressed miRNA in transverse aortic constriction-induced hypertrophy and physiological hypertrophy of the neonatal heart. A density heatmap comparing the distribution of the $\log 2$ values of miRNA differentially expressed in the hearts of mice subjected to TAC/sham (x-axis) versus those of neonatal/adult hearts (y-axis). Red is a region containing data points more than expected; blue is a region containing data points less than expected (scale, right). The heatmap shows a majority of miRNA that are similarly downregulated (red, lower left quadrant) or upregulated (red, upper right quadrant) in both the hypertrophy/sham and neonatal/adult hearts. The red in the upper left quadrant represents miRNA that were upregulated during hypertrophy but were lower in the neonatal vs. adult

have attracted much attention. MiRNAs impact protein expression in a way similar to a transcription factor, that is by effecting a specific increase or decrease in the translation product. For many one and for one many, characterizes how both these molecules recognize their targets, while fundamentally diverging in the underlying mechanism of function. Whereas transcription factors regulate mRNA synthesis of a gene through binding its promoter, miRNA directly bind to a specific sequence within the 3'UTR of an mRNA and inhibit protein translation and/or induced mRNA degradation (Fig. 2). In general, when a miRNA is attached, translation is off, and vice versa, thus, functioning as a molecular switch.

What we can extrapolate from this comparison is that miRNAs can accomplish their function in a more direct and, thus, a more rapid fashion, relative to transcription factors. Transcriptional regulation not only involves mRNA synthesis, but most often requires DNA remodeling as well. Additionally, in the event that inhibition of protein synthesis is the objective, the effect would be delayed by as long as the decay time of the preexisting mRNA. Overall, transcriptional regulation is evidently more energy- and time-consuming relative to miRNA regulation. This leads us to propose that deregulation of miRNA would allow the cell to respond to an 


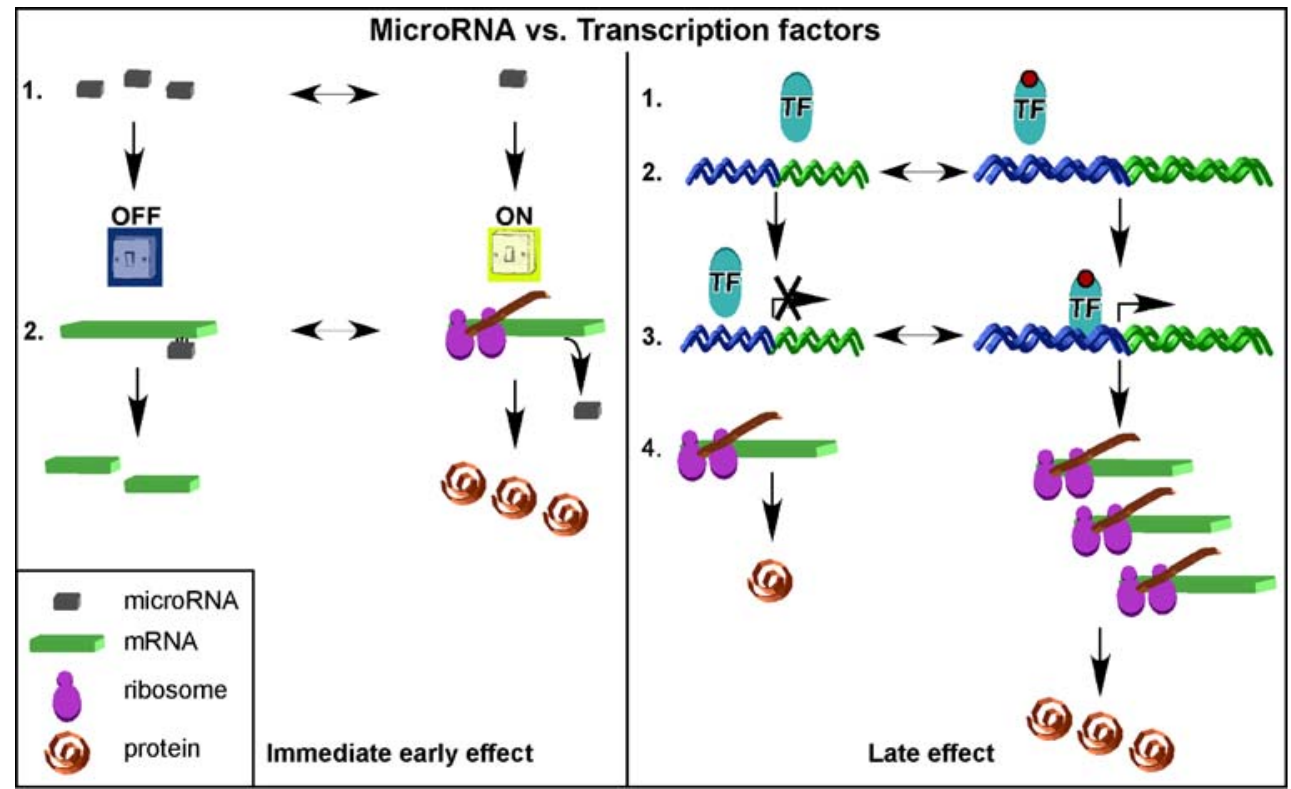

Fig. 2 Comparison of posttranscriptional regulation by miRNA vs. transcriptional regulation of gene expression. 1 Levels of miRNA are posttranscriptionally regulated. 2 This results in a miRNA attachment to the 3'UTR of a gene and inhibition of translation or mRNA degradation, or its detachment and activation of translation, thus, acting as a molecular switch that could result in an immediate early effect in

response to an incoming signal. On the other hand, transcriptional regulation requires: 1 DNA remodeling; 2 attachment or detachment of the transcription factor; and 3 inhibition or activation of RNA synthesis, before an effect on 4 protein levels is observed, thus, resulting in a relatively late response to an incoming signal

incoming signal without delay. One caveat to this theory, though, is that miRNA levels themselves have to be altered before eliciting a change in a gene's translation. It has been generally assumed that miRNA are mainly regulated by transcriptional events, which would negate the possibility of their immediate response to a cell signal. Recently, though, data has been accumulating regarding posttranscriptional regulation of miRNA, as will be discussed below.

\section{MiRNA as Immediate-Early Responders to Cellular Stimuli}

Our recent data demonstrate that some miRNA are deregulated relatively early in response to a stimulus, which provides a new prospect for therapeutics. We have reported that the heart responds to pressure overload by immediately downregulating miR-1, prior to any obvious change in gene expression, heart size, or function. Downregulation of miR-1 implies that the translation of its targets will be uninhibited. Although we have not ruled out transcriptional regulation of miR-1, we have discovered that the RNA binding and hydrolyzing protein Ras-GAP SH3-binding protein (G3BP) binds to the miR-1 precursor through a conserved consensus sequence. The significance of this finding was tested by measuring the effect of overexpression or knockout of G3BP on miR-1 in myocytes, the results of which showed downregulation or upregulation of miR-1, respectively (D. Sayed

and M. Abdellatif, unpublished data). G3BP itself is regulated by phosphorylation. The results provide a mechanism whereby miR-1 is rapidly downregulated, while circumventing a need for transcriptional regulation. This intimates that miR-1, has the capacity to function as one of the first responders to hypertrophic stimuli, and is a cause rather than an effect of cardiac hypertrophy. Consequently, we speculated that miR-1 target genes must play a fundamental role in initiating cardiac hypertrophy. In accordance, we identified many predicted targets that fit that description and have confirmed a few by Western blotting. Some of those genes are listed above, but the most intriguing is eIF4E. EIF4e is upregulated during cardiac hypertrophy in a mouse model, which is inhibited if downregulation of miR-1 is normalized by an exogenous construct, as seen in cultured rat neonatal myocytes (Fig. 3). Since eIF4E is the limiting factor in translation initiation of all capped mRNA, it was not surprising that all luciferase reporter constructs, with or without the miR-1 target site in the 3'UTR, were dramatically inhibited in the presence of excess miR-1, making it difficult to distinguish its direct from indirect targets (D. Sayed and M. Abdellatif, unpublished data). EIF4e is, conceivably, the Achilles heel of cardiac hypertrophy, by reason that it is the limiting factor in protein synthesis, which elicits an increase in cell size that defines hypertrophy. This further emphasizes a role for miR-1 in initiating cardiac hypertrophy.

There is accumulating evidence that miRNAs are regulated by posttranscriptional mechanisms. First demon- 


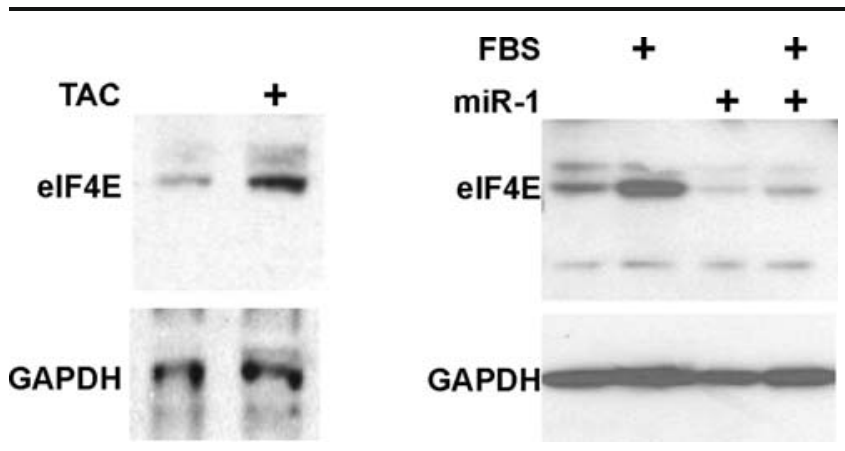

Fig. 3 Eukaryotic initiation factor 4E (eIF4E) is upregulated during cardiac myocyte hypertrophy and is inhibited by miR-1. Left panels Western blot analysis of protein extracted from pressure overloaded (TAC), hypertrophied, mouse hearts (where marked by a + sign above the Western blot lane), or a sham control (unmarked lane), using an eIF4E antibody (upper panel) or a glyceraldehyde 3-phosphate dehydrogenase (GAPDH) antibody for an internal control (lower panel). Right panels rat cardiac myocytes were cultured in fetal bovine serum (FBS)-free medium. Cells were then stimulated with $10 \%$ FBS for $24 \mathrm{~h}$ to induce hypertrophy, in the absence or presence of excess miR-1 that was delivered by recombinant adenovirus (where marked by + signs above the Western blot lanes). Total protein was extracted and analyzed by Western blotting as indicated above

strated by Thomson et al, where they showed that there is little or no correlation between primary and mature miRNA, plausibly due to selective processing by Drosha in both normal and cancer tissue [15]. More recently, it was discovered that Lin-28 selectively binds to and inhibits the processing of primary let-7 miRNA $[16,17]$. Furthermore, miRNAs are subject to posttranscriptional editing, where adenosine is converted to inosine. Luciano et al were the first to notice that premature miR-22 is edited at multiple sites [18]. It is estimated that $\sim 6 \%$ of human miRNA are edited [19]. Importantly, some of the edited sites are within the 'seed' region and result in reassignment of targets [20]. This demonstrates the extensiveness of posttranscriptional regulation of miRNA as a mechanism that would elicit a rapid change in their level or targeting capacity in response to a cell signal.

\section{miRNA vs. mRNA for Therapeutic Targeting}

As well established, a single protein does not independently fulfill a cellular function, but is part of a consortium of genes with distinct, but also overlapping, roles that ensure perpetuation of function. For example, we would argue that while upregulation of eIF4E by way of downregulation of miR-1 is an essential step in initiating protein synthesis, other targets are necessary for maximal protein synthesis. For example, RasGAP is required for activating RNA polymerase II and subsequently mRNA synthesis [21]. Since, an increase in protein synthesis would be limited by mRNA availability, it becomes clear why upregulation of RasGAP is equally important for maximizing protein synthesis. Accordingly, we predict that other growth-related targets of miR-1 collaborate with eIF4E to achieve complete hypertrophic growth. From that prospective, it would be more effective to inhibit hypertrophy by normalizing miR-1 versus any single mRNA target. Similarly, it was more effective to inhibit proliferation by simultaneously rather than individually inhibiting the cell cycle-related miR-16 targets [22]. This demonstrates that miRNA have the potential to serve as more powerful therapeutic targets than would individual genes.

Although we have a wealth of information regarding the differentially expressed miRNA in cardiac hypertrophy and failure [4, 23-27], we know little about their targets, functions, or cause-effect relationship. Besides miR-1 that we have discussed above, the downregulation of miR-133a was proven sufficient for induction of hypertrophy [24]. Interestingly, both these miRNAs are muscle-enriched. On the other hand, the mature form of cardiac-specific miR-208 remains unaltered in either human failing hearts or during cardiac hypertrophy in wild type mice [28], thus, should have no consequence on its targets' expression or therapeutic value. Another potential therapeutic target is miR195 , which is upregulated during cardiac hypertrophy and induces cardiac failure in a transgenic mouse model [23]. It is worth noting that miR-195, among a few others, deviated from the characteristic rewind to the neonatal pattern seen during hypertrophy. These few miRNA are represented in the upper left quadrant of the heatmap in Fig. 1. The changes in these miRNA might distinguish pathological from physiological growth. Identifying the targets and functions of these miRNA would help validate this idea and their potential for therapeutic targeting.

On the other hand, miR-21 plays a positive role during growth by inhibiting apoptosis as demonstrated in cancerous cells $[29,30,31]$ and inducing cell invasion, but not proliferation, through inhibition of programmed cell death 4 (Pdcd4) [32]. In human colorectal cancer the levels of miR21 positively correlated with the development of metastasis but not tumor size [33]. In the heart, we have shown that miR-21 is upregulated in parallel with the increase in cardiac mass, but starts declining upon the onset of cardiac dysfunction and dilatation [34]. Transgenic mouse lines overexpressing miR-21, $\sim 10$ and 30 -fold in excess of endogenous, do not exhibit an overt phenotype, where cardiac size, histology, and all functional parameters are normal (D. Sayed and M. Abdellatif, unpublished results). But upon closer investigation, we determined that miR-21 induced lateralization of connexin $43(\mathrm{Cx} 43)$ that is otherwise strictly localized to the intercalated discs (Fig. 4). This is similar to the redistribution of $\mathrm{Cx} 43$ seen in peri-infarct zones [35] and during adaptive hypertrophy [36, 37], thought to enhance conduction and decrease arrhythmias. At the onset of cardiac failure, though, this effect is reversed, 


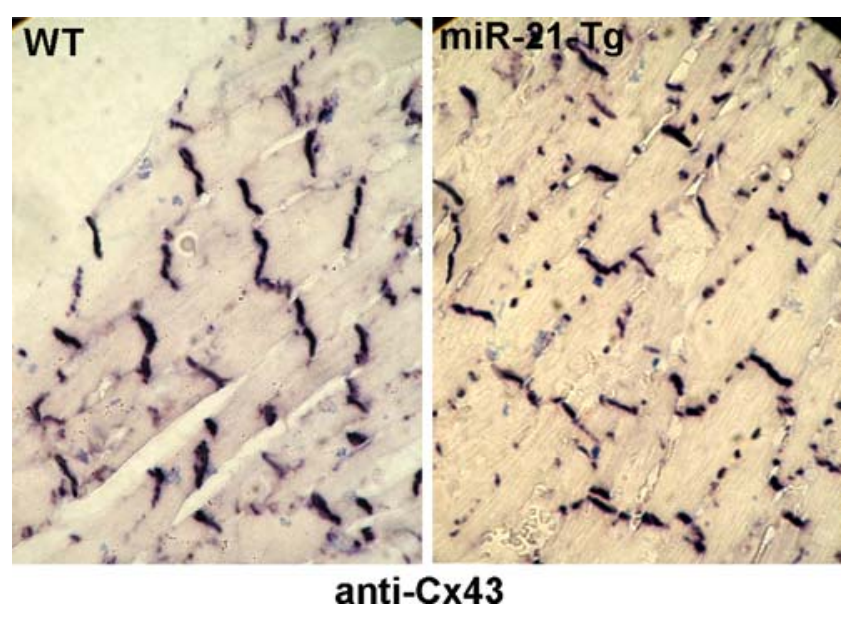

Fig. 4 Lateralization of $\mathrm{Cx} 43$ in miR-21 transgenic hearts. Crosssections of hearts from 7-month-old males, wild type and miR-21, cardiac-specific, transgenic mice, immunostained with anti-Cx43 (dark purple)

as is the upregulation of miR-21. Thus, this would be a good example of a miRNA that would benefit from gene replacement therapy during cardiac failure.

\section{Tools for Modulating miRNA Levels}

MiRNA can be either upregulated or downregulated during a disease and will accordingly require different approaches for normalizing its levels. In this section we describe the available tools and techniques that have been developed for those purposes.

MiRNA Specific Antisense oligonucleotides Antisense oligodeoxynucleotides are widely used as a tool for targeting and inhibiting mRNA translation. Various backbone and 2'-sugar modifications were introduced to enhance their stability and cellular uptake. For example, phosphorothioate modifications, where a nonbridging oxygen within the internucleoside bond is replaced by a sulfur atom, render oligonucleotides more resistant to nuclease activity, thus, prolonging their half-life [38]. Moreover, such modified oligonucleotides were found to be highly bound to plasma proteins, which delayed their urinary excretion [39]. It should be noted, though, that the phosphorothioate-modified linkers decreased the affinity to the complementary sequence. Also, it was found that 2'$\mathrm{O}$-sugar modifications are essential for an oligonucleotide's effectiveness, where those uniformly modified with 2'O-methoxyethyl (2'-O-MOE) were much more effective than those with 2'-O-methyl (2'-O-Me) [40]. But at high doses only $2^{\prime}-\mathrm{O}-\mathrm{Me}$ induced significant toxicity in cultured Hela cells. These oligonucleotides appear to enter the cells via scavenger receptors and when injected in vivo become highly concentrated in the liver, kidneys, and bone marrow [41].
Different versions of modified and cholesterol conjugated antisense miRNAs were applied in vivo with great success. Hutvagner et al. were the first to report that 2'O-methyl oligonucleotides successfully inhibited miRNA let-7 function in Drosophila [42], while Esau et al. observed enhanced inhibition of liver miR-122 in mice by using 2'O-MOE phosphorothioate modified oligodeoxynucleotides [43]. In the latter case, the minimum effective dose tested for lowering cholesterol was $12.5 \mathrm{mg} / \mathrm{kg}(2 \times /$ week for 4 weeks), although, miR-122 was further reduced at higher doses. Similarly, injection of 2'-O-methyl, phosphorothioate, 5'-cholesterol-conjugated antisense miR-122 modified oligonucleotides "antagomirs", inhibited miR-122 in the liver [44]. In addition, the use of unusually high doses $(3 \times 80 \mathrm{mg} / \mathrm{kg})$ of the antagomirs successfully knocked down miR-16 in other organs including lung, kidney, heart, intestine, fat, skin, bone marrow, muscle, ovaries, and adrenals. The utility of antagomirs was further confirmed in the heart by knockdown of miR-133a, which demonstrated its role in inducing hypertrophy [24]. These antagomirs appear to function by binding to the mature miRNA within the cytoplasmic compartment, but not in P-bodies, and induces its degradation [45].

MiRNA Sponges As the name implies, this construct is designed to absorb cellular miRNA, but unlike the antisense oligonucleotides, it does not induce significant degradation of the target [46]. There are 2 different designs, one where multiple specific miRNA complimentary sites are incorporated in the 3'UTR of a gene, such as green florescence

\section{miR eraser U6 promoter (anti-miR)X2-polyT}

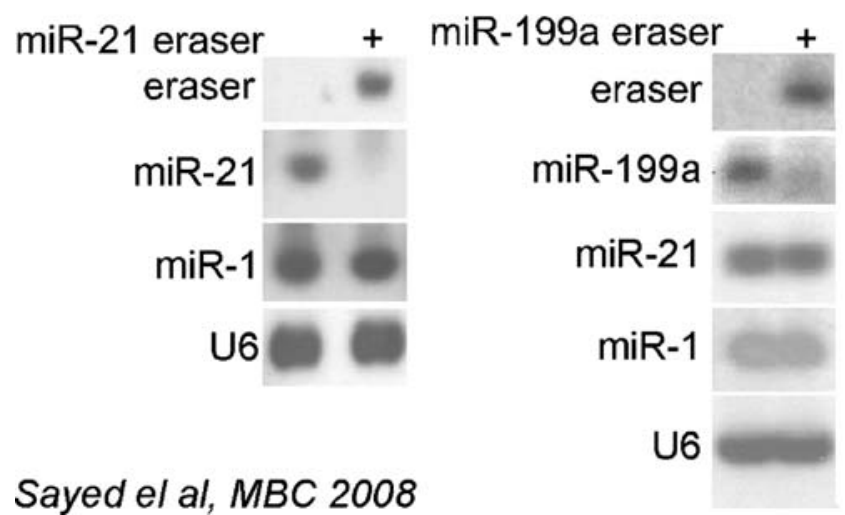

Fig. 5 'MiRNA erasers' induce downregulation of endogenous miRNA. A schematic of the miR eraser, which is a tandem repeat of a specific miRNA antisense sequence, expressed from an RNA polymerase III-dependent promoter such as U6. The panels below are Northern blots of RNA extracted from cardiocytes that were infected with 10-20 moi of the miR-21 or miR-199a erasers for $48 \mathrm{~h}$ $(n=3)$. The + sign identifies the sample lane which received the eraser and the labels on the left define the observed signals [34]. The figure is a reproduction from ref. [43] 
protein expressed from an RNA polymerase II-dependent promoter, which can also be used as a reporter. The alternative design just includes the multiple specific miRNA complimentary sites flanked by stabilizing stem-loop sequences, expressed by an RNA polymerase III-dependent promoter. The study showed that those constructs successfully antagonized miRNA function in cultured cells. On the other hand, Brown et al. used a similar design, with multiple miRNA target sites in the 3'UTR of a transgene, for the purpose of suppressing transgene expression in cells expressing the corresponding miRNA [47]. In these experiments, the authors showed that this sponge-like construct did not inhibit the function of the endogenous miRNA. Thus, we realize that more experiments are necessary for validating the function of the sponges. Another aspect of this method that still remains to be addressed, is the fate of the accumulated sponge RNAmiRNA duplexes and their effect on cell function.

MiRNA Erasers As disclosed by the name, erasers induce almost complete elimination of endogenous miRNA (Fig. 5) [34]. They employ the same design concept as that of the sponges', which includes specific miRNA complimentary sites expressed by an RNA polymerase III-dependent promoter, but differ in some aspects: inclusion of only two miRNA binding site vs. four to seven for the sponge and the absence of the flanking stem-loops. This renders the expressed eraser construct much shorter (40-44 nt) than the corresponding sponge $(<300 \mathrm{nt})$. In addition, the erasers were delivered to cultured cells via recombinant adenoviruses. It is conceivable that these structural differences accounted for the mechanistic difference between the two constructs. The erasers could be useful in generating transgenic animal models, as a convenient alternative to genomic knockout of miRNA. Moreover, they would provide a solution for knocking out miRNA that exist within exons, such as miR-21, or others that are expressed from more than one gene. In addition, they could serve as effective tools for in vivo therapeutic targeting of miRNA.

MiRNA replacement strategies From the abundance of information that has been reported regarding miRNA expression patterns in various diseases, it is clear that downregulated miRNA are represented as frequently as those upregulated. Thus, to address their functional significance or to target them for replacement therapy, a couple of tools have been developed. One was dubbed miRNA "mimics" or "mimetics". This entails delivering a duplex including the miRNA and its compliment. This design, versus the single-stranded mature miRNA, was necessary for efficient incorporation of the mature miRNA into the RISC complex. The approach has been successfully tested in cultured cells $[48,49]$ and the same strategies described above for delivery of oligonucleotides would apply here. Alternatively, miRNA were also successfully expressed from their genomic or stem-loop precursor sequence by constitutive or tissue specific promoters, in tissue culture and transgenic mice models [4, 23, 34].

\section{Exploiting Endogenous miRNA to Restrict Transgene Expression}

Tissue-targeted transgene expression is a desirable feature for gene therapy. While this is achievable through tissuespecific promoters, in many cases this option does not readily lend itself, such as in undifferentiated normal or cancerous cells. Recently, Brown et al. showed that by introducing selective miRNA target sites into the 3'UTR of a transgene it is possible to suppress its expression in cells that express the corresponding miRNA [47]. As proof of principle, the authors showed that a GFP construct containing the human embryonic stem cell (hES)-specific miR-372 target site, was only expressed during differentiated states where miR-372 was downregulated. This could provide a means to segregate differentiated from undifferentiated ES cells. Similarly, one can envision a design whereby an apoptotic gene can be delivered to a tumor, while avoiding its expression in major organs such as the heart, brain, and liver, by introducing the combination of miR-1, miR-124, and miR-122 target sites in the 3'UTR of the transgene.

\section{Concluding Remarks}

Since their discovery in mammalian cells, there has been tremendous progress in the understanding of miRNA and their expression patterns in health and disease. As presented above, these molecules have the potential to become more effective therapeutic targets than single mRNA molecules. But if we want to fully exploit them for diagnostic, prognostic, or therapeutic purposes, we need to learn more about the function of each individual miRNA and its range of targets.

Open Access This article is distributed under the terms of the Creative Commons Attribution Noncommercial License which permits any noncommercial use, distribution, and reproduction in any medium, provided the original author(s) and source are credited.

\section{References}

1. Gygi, S. P., Rochon, Y., Franza, B. R., \& Aebersold, R. (1999). Correlation between protein and mRNA abundance in yeast. Molecular and Cellular Biology, 19(3), 1720-1730. 
2. Beyer, A., Hollunder, J., Nasheuer, H. P., \& Wilhelm, T. (2004). Post-transcriptional expression regulation in the yeast Saccharomyces cerevisiae on a genomic scale. Molecular \& Cellular Proteomics, 3(11), 1083-1092.

3. Wang, Y., Liu, C. L., Storey, J. D., Tibshirani, R. J., Herschlag, D., \& Brown, P. O. (2002). Precision and functional specificity in mRNA decay. Proceedings of the National Academy of Sciences of the United States of America, 99(9), 5860-5865.

4. Sayed, D., Hong, C., Chen, I. Y., Lypowy, J., \& Abdellatif, M. (2007). MicroRNAs play an essential role in the development of cardiac hypertrophy. Circulation Research, 100(3), 416-424.

5. John, B., Enright, A. J., Aravin, A., Tuschl, T., Sander, C., \& Marks, D. S. (2004). Human MicroRNA targets. PLoS Biology, 2 (11), 1862-1879.

6. Farh, K. K., Grimson, A., Jan, C., Lewis, B. P., Johnston, W. K., Lim, L. P., et al. (2005). The widespread impact of mammalian MicroRNAs on mRNA repression and evolution. Science, 310 (5755), 1817-1821.

7. Lim, L. P., Lau, N. C., Garrett-Engele, P., Grimson, A., Schelter, J. M., Castle, J., et al. (2005). Microarray analysis shows that some microRNAs downregulate large numbers of target mRNAs. Nature, 433(7027), 769-773.

8. Johnatty, S. E., Dyck, J. R., Michael, L. H., Olson, E. N., \& Abdellatif, M. (2000). Identification of genes regulated during mechanical load-induced cardiac hypertrophy. Journal of Molecular and Cellular Cardiology, 32(5), 805-815.

9. Lagos-Quintana, M., Rauhut, R., Yalcin, A., Meyer, J., Lendeckel, W., \& Tuschl, T. (2002). Identification of tissue-specific microRNAs from mouse. Current Biology, 12(9), 735-739.

10. Zhao, Y., Samal, E., \& Srivastava, D. (2005). Serum response factor regulates a muscle-specific microRNA that targets Hand2 during cardiogenesis. Nature, 436(7048), 214-220.

11. Sokol, N. S., \& Ambros, V. (2005). Mesodermally expressed Drosophila microRNA-1 is regulated by Twist and is required in muscles during larval growth. Genes \& Development, 19(19), 2343-2354.

12. Zhao, Y., Ransom, J. F., Li, A., Vedantham, V., von Drehle, M., Muth, A. N., et al. (2007). Dysregulation of cardiogenesis, cardiac conduction, and cell cycle in mice lacking miRNA-1-2. Cell, 129 (2), 303-317.

13. Care, A., Catalucci, D., Felicetti, F., Bonci, D., Addario, A., Gallo, P., et al. (2007). MicroRNA-133 controls cardiac hypertrophy. Natural Medicines, 13(5), 613-618.

14. Yang, B., Lin, H., Xiao, J., Lu, Y., Luo, X., Li, B., et al. (2007). The muscle-specific microRNA miR-1 regulates cardiac arrhythmogenic potential by targeting GJA1 and KCNJ2. Natural Medicines, 13(4), 486-491.

15. Thomson, J. M., Newman, M., Parker, J. S., Morin-Kensicki, E. M., Wright, T., \& Hammond, S. M. (2006). Extensive posttranscriptional regulation of microRNAs and its implications for cancer. Genes and Development, 20(16), 2202-2207.

16. Newman, M. A., Thomson, J. M., \& Hammond, S. M. (2008). Lin-28 interaction with the Let-7 precursor loop mediates regulated microRNA processing. $R N A, 14(8), 1539-1549$.

17. Viswanathan, S. R., Daley, G. Q., \& Gregory, R. I. (2008). Selective blockade of microRNA processing by Lin28. Science, 320(5872), 97-100.

18. Luciano, D. J., Mirsky, H., Vendetti, N. J., \& Maas, S. (2004). RNA editing of a miRNA precursor. RNA, 10(8), 1174-1177.

19. Blow, M. J., Grocock, R. J., van Dongen, S., Enright, A. J., Dicks, E., Futreal, P. A., et al. (2006). RNA editing of human microRNAs. Genome Biology, 7(4), 4.

20. Kawahara, Y., Zinshteyn, B., Sethupathy, P., Iizasa, H., Hatzigeorgiou, A. G., \& Nishikura, K. (2007). Redirection of silencing targets by adenosine-to-inosine editing of miRNAs. Science, 315(5815), 1137-1140.
21. Abdellatif, M., Packer, S. E., Michael, L. H., Zhang, D., Charng, M. J., \& Schneider, M. D. (1998). A Ras-dependent pathway regulates RNA polymerase II phosphorylation in cardiac myocytes: implications for cardiac hypertrophy. Molecular and Cellular Biology, 18(11), 6729-6736.

22. Linsley, P. S., Schelter, J., Burchard, J., Kibukawa, M., Martin, M. M., Bartz, S. R., et al. (2007). Transcripts targeted by the microRNA-16 family cooperatively regulate cell cycle progression. Molecular and Cellular Biology, 27(6), 22402252 .

23. van Rooij, E., Sutherland, L. B., Liu, N., Williams, A. H., McAnally, J., Gerard, R. D., et al. (2006). A signature pattern of stress-responsive microRNAs that can evoke cardiac hypertrophy and heart failure. Proceedings of the National Academy of Sciences of the United States of America, 103(48), 18255-18260.

24. Care, A., Catalucci, D., Felicetti, F., Bonci, D., Addario, A., Gallo, P., et al. (2007). MicroRNA-133 controls cardiac hypertrophy. Natural Medicines, 13(5), 613-618.

25. Callis, T. E., Chen, J. F., \& Wang, D. Z. (2007). MicroRNAs in skeletal and cardiac muscle development. DNA and Cell Biology, 26(4), 219-225.

26. Tatsuguchi, M., Seok, H. Y., Callis, T. E., Thomson, J. M., Chen, J. F., Newman, M., et al. (2007). Expression of microRNAs is dynamically regulated during cardiomyocyte hypertrophy. Journal of Molecular and Cellular Cardiology, 42(6), 1137-1141.

27. Cheng, Y., Ji, R., Yue, J., Yang, J., Liu, X., Chen, H., et al. (2007). MicroRNAs are aberrantly expressed in hypertrophic heart: do they play a role in cardiac hypertrophy? American Journal of Pathology, 170(6), 1831-1840.

28. van Rooij, E., Sutherland, L. B., Qi, X., Richardson, J. A., Hill, J., \& Olson, E. N. (2007). Control of stress-dependent cardiac growth and gene expression by a MicroRNA. Science, 316(5824), 575-579.

29. Chan, J. A., Krichevsky, A. M., \& Kosik, K. S. (2005). MicroRNA-21 is an antiapoptotic factor in human glioblastoma cells. Cancer Research, 65(14), 6029-6033.

30. Meng, F., Henson, R., Wehbe-Janek, H., Ghoshal, K., Jacob, S. T., \& Patel, T. (2007). MicroRNA-21 regulates expression of the PTEN tumor suppressor gene in human hepatocellular cancer. Gastroenterology, 133(2), 647-658.

31. Loffler, D., Brocke-Heidrich, K., Pfeifer, G., Stocsits, C., Hackermuller, J., Kretzschmar, A. K., et al. (2007). Interleukin-6 dependent survival of multiple myeloma cells involves the Stat3mediated induction of microRNA-21 through a highly conserved enhancer. Blood, 110(4), 1330-1333.

32. Asangani, I. A., Rasheed, S. A., Nikolova, D. A., Leupold, J. H., Colburn, N. H., Post, S., et al. (2007). MicroRNA-21 (miR-21) post-transcriptionally downregulates tumor suppressor Pdcd4 and stimulates invasion, intravasation and metastasis in colorectal cancer. Oncogene, 27, 2128-2136.

33. Slaby, O., Svoboda, M., Fabian, P., Smerdova, T., Knoflickova, D., Bednarikova, M., et al. (2008). Altered expression of miR-21, miR-31, miR-143 and miR-145 is related to clinicopathologic features of colorectal cancer. Oncology, 72(5-6), 397-402.

34. Sayed, D., Rane, S., Lypowy, J., He, M., Chen, I.Y., Vashistha, H., et al. (2008). MicroRNA-21 targets sprouty2 and promotes cellular outgrowths. Molecular Biology of the Cell, 19(8), 3272-3282.

35. Smith, J. H., Green, C. R., Peters, N. S., Rothery, S., \& Severs, N. J. (1991). Altered patterns of gap junction distribution in ischemic heart disease. An immunohistochemical study of human myocardium using laser scanning confocal microscopy. American Journal of Pathology, 139(4), 801-821.

36. Emdad, L., Uzzaman, M., Takagishi, Y., Honjo, H., Uchida, T., Severs, N. J., et al. (2001). Gap junction remodeling in hypertrophied left ventricles of aortic-banded rats: prevention by angiotensin II type 1 receptor blockade. Journal of Molecular and Cellular Cardiology, 33(2), 219-231. 
37. Uzzaman, M., Honjo, H., Takagishi, Y., Emdad, L., Magee, A. I., Severs, N. J., et al. (2000). Remodeling of gap junctional coupling in hypertrophied right ventricles of rats with monocrotaline-induced pulmonary hypertension. Circulation Research, 86(8), 871-878.

38. Milligan, J. F., Matteucci, M. D., \& Martin, J. C. (1993). Current concepts in antisense drug design. Journal of Medicinal Chemistry, 36(14), 1923-1937.

39. Geary, R. S., Watanabe, T. A., Truong, L., Freier, S., Lesnik, E. A., Sioufi, N. B., et al. (2001). Pharmacokinetic properties of 2'-O-(2-methoxyethyl)-modified oligonucleotide analogs in rats. Journal of Pharmacology and Experimental Therapeutics, 296(3), 890-897.

40. Davis, S., Lollo, B., Freier, S., \& Esau, C. (2006). Improved targeting of miRNA with antisense oligonucleotides. Nucleic Acids Research, 34(8), 2294-2304.

41. Bijsterbosch, M. K., Manoharan, M., Rump, E. T., De Vrueh, R. L., van Veghel, R., Tivel, K. L., et al. (1997). In vivo fate of phosphorothioate antisense oligodeoxynucleotides: predominant uptake by scavenger receptors on endothelial liver cells. Nucleic Acids Research, 25(16), 3290-3296.

42. Hutvagner, G., Simard, M. J., Mello, C. C., \& Zamore, P. D. (2004). Sequence-specific inhibition of small RNA function. PLoS Biology, 2(4), 24
43. Esau, C., Davis, S., Murray, S. F., Yu, X. X., Pandey, S. K., Pear, M., et al. (2006). miR-122 regulation of lipid metabolism revealed by in vivo antisense targeting. Cell Metabolism, 3(2), 87-98.

44. Krutzfeldt, J., Rajewsky, N., Braich, R., Rajeev, K. G., Tuschl, T., Manoharan, M., et al. (2005). Silencing of microRNAs in vivo with 'antagomirs'. Nature, 438(7068), 685-689.

45. Krutzfeldt, J., Kuwajima, S., Braich, R., Rajeev, K. G., Pena, J., Tuschl, T., et al. (2007). Specificity, duplex degradation and subcellular localization of antagomirs. Nucleic Acids Research, 35 (9), 2885-2892.

46. Ebert, M. S., Neilson, J. R., \& Sharp, P. A. (2007). MicroRNA sponges: competitive inhibitors of small RNAs in mammalian cells. Nature Methods, 4(9), 721-726.

47. Brown, B. D., Gentner, B., Cantore, A., Colleoni, S., Amendola, M., Zingale, A., et al. (2007). Endogenous microRNA can be broadly exploited to regulate transgene expression according to tissue, lineage and differentiation state. Nature Biotechnology, 25(12), 1457-1467.

48. Ford, L. P. (2006). Using synthetic miRNA mimics for diverting cell fate: a possibility of miRNA-based therapeutics? Leukemia Research, 30(5), 511-513.

49. Guimaraes-Sternberg, C., Meerson, A., Shaked, I., \& Soreq, H. (2006). MicroRNA modulation of megakaryoblast fate involves cholinergic signaling. Leukemia Research, 30(5), 583-595. 